\title{
A Scattered Field Formulation of the Time-Domain Radial Point Interpolation Method using Radial Perfectly Matched Layers
}

\author{
Thomas Kaufmann ${ }^{\# 1}$, Christophe Fumeaux \#2 \\ \# School of Electrical \& Electronic Engineering, \\ The University of Adelaide, Adelaide, South Australia 5005, Australia \\ ${ }^{1}$ thomaskadeleceng. adelaide.edu.au \\ ${ }^{2}$ cfumeauxdeleceng. adelaide.edu.au
}

\begin{abstract}
Meshless methods are a new type of numerical schemes in computational electromagnetics, combining the advantages of conformal unstructured modeling with the flexibility of a node distribution without an explicit mesh topology. A scattered field formulation of the meshless Radial Point Interpolation Method (RPIM) is introduced for efficient simulation of metallic structures. Scattering problems generally result in spherically radiated wavefronts, hence truncating the computational domain with locally radial perfectly matched layers (PML) appears more effective than with classical uniaxial PML. Therefore, such problems can be modelled using less memory and shorter computation times with spherical or cylindrical PML. The scattered field RPIM formulation with radial PML is verified in a classical scattering problem from a perfectly conducting cylinder. A comparison with the analytical Mie solution shows fast convergence rates which are indicative of low reflections from the PML boundary. A convergence analysis and a study on the PML thickness demonstrates how to extend the limits of the PML formulation.
\end{abstract}

\section{INTRODUCTION}

Meshless methods are an interesting new field in computational electromagnetics, which combines the versatility of unstructured discretization with the simplicity of a node-based scheme. Instead of a mesh topology, electric and magnetic fields are stored at arbitrarily distributed node locations. This allows conformal modeling of complex structures without the overhead associated with an explicitly defined mesh structure. Using sophisticated local basis functions, high accuracy can be achieved at a relatively low computational cost.

Among the various existing formulations of meshless methods, the Radial Point Interpolation Method (RPIM) for timedomain electromagnetics is the focus of this paper. This method has been first introduced in a two-dimensional setting in [1] and extended to 3D in [2]. To solve some observed long-time stability issues [3], an unconditionally stable formulation has been presented in [4]. An alternative approach using Laguerre polynomials to discretize the time variable has been formulated in [5]. To simulate open radiation and scattering problems, perfectly matched layers (PML) have been introduced in [6]. The most recent developments of the RPIM have been summarized in [7].

In this paper, a scattered field formulation for the simulation of scattering from metallic objects is presented using radial
PML [8]. The scattered field formulation, in contrast to the total field/scattered field formulation, imposes the conditions of an incoming plane wave on perfectly conducting boundaries. It has the advantage of shorter simulation times and it reduces the amount of power to be absorbed by the PML. The method is verified by comparison of the results to the classical Mie solution for the scattering from a perfectly conducting cylinder.

\section{Radial Point Interpolation Method}

The RPIM scheme has been introduced initially for mechanical problems as an extension of the point interpolation method with radial basis functions (RBF) [9] in a Galerkin formulation. The local interpolation scheme was then applied to the solution of Maxwell's equations [1]. The interpolation of a field value in one given point is based on the field values $\mathbf{u}^{e}$ at surrounding node locations inside a local support domain, usually enclosing $N=8-12$ neighboring nodes (in $2 \mathrm{D}$ ). In a point-matching scheme, a combination of radial and polynomial basis functions are evaluated on the neighbor locations. This results in a symmetrical positive-definite moment matrix

$$
\mathbf{G}=\left(\begin{array}{cc}
\mathbf{R} & \mathbf{P} \\
\mathbf{P}^{T} & \mathbf{0}
\end{array}\right)
$$

with the RBFs evaluated in $\mathbf{R}_{i, j}=r_{i}\left(\mathbf{x}_{j}\right)$ and the first-order polynomial basis functions as $\mathbf{P}_{i,:}=\left[\begin{array}{lll}1 & x_{i} & y_{i}\end{array}\right]$. Gaussian basis functions are a common choice for the RBFs centered around node location $\mathbf{x}_{i}=\left(x_{i}, y_{i}\right)$

$$
r_{i}\left(\mathbf{x}_{n}\right)=\exp \left(-\alpha_{c}\left(\frac{\mathbf{x}_{i}-\mathbf{x}_{n}}{d_{c}}\right)^{2}\right)
$$

with a shape parameter $\alpha_{c}$ and the average node distance $d_{c}$. Eventually the interpolation of a field value at the position $\mathbf{x}=x, y$ can be calculated through the inversion of a small local matrix $\mathbf{G}$ as

$$
u(\mathbf{x}) \approx\left[r_{1}(\mathbf{x}), \ldots, r_{N}(\mathbf{x})\right] \mathbf{G}^{-1} \mathbf{u}^{e}=\mathbf{\Psi}(\mathbf{x}) \mathbf{u}^{e},
$$

i.e. the interpolation is carried out as a linear combination of the $N$ surrounding field values. This interpolation fulfills the delta property, i.e. the values at the node locations are reconstructed perfectly. As a consequence, explicit time stepping 
schemes can be implemented without the need of a matrix inversion during the time iteration.

\section{SCATTERED FIELD FORMULATION}

The scattered field formulation for metallic structures is adapted from [10], assuming an incoming broadband plane wave signal. The total electric field $E_{\text {total }}$ in the computational domain is determined as

$$
E_{\text {total }}=E_{\text {inc }}+E_{\text {scat }} .
$$

The incident plane wave $E_{i n c}$ is a known analytical function expressed as

$$
\begin{array}{r}
E_{\text {inc }}=\exp \left(-\left(\frac{t-\left(x-x_{0}\right) / c_{0}-4 \sigma_{0}}{\sqrt{(2) \sigma_{0}}}\right)^{2}\right) \\
\sin \left(\omega\left(t-\left(x-x_{0}\right) / c_{0}\right)-4 \sigma_{0}\right)
\end{array}
$$

for propagation in $x$-direction with the time variable $t$ and position $x$. The width of the pulse is determined by $\sigma_{0}$ and a spatial offset $x_{0}$ allows to place the beginning of the pulse at the first occurrence of the metallic structure. On the metallic objects, perfectly electrically conducting (PEC) boundary conditions apply, which provides the necessary condition for numerical computation of the scattered field $E_{\text {scat }}$. In mathematical form the total and scattered field formulation of the transverse electric field are defined as follows

$$
\begin{array}{ll}
E_{P E C}=0 & \text { (total field formulation) } \\
E_{P E C}=-E_{\text {inc }} & \text { (scattered field formulation). }
\end{array}
$$

Therefore, in the scattered field formulation, the source conditions are applied at each point on the perfectly conducting object, both in space and time, and the solver yields the scattered field.

\section{Radial Perfectly Matched LAyers}

PML [11] are an artificial type of absorbing boundary condition that operate on the truncating boundary of a computational domain. The electromagnetic properties inside this layer are determined such that the material is matched to the surrounding medium (often free space). The electric and magnetic conductivities of the material are usually progressively increased towards the outer boundary in a polynomial profile to minimize numerical reflections. The original formulation exploited the uniaxial grid of the finite-difference time-domain (FDTD) method and therefore was placed along coordinate axes. In many scattering and radiation problems though, spherical waves have to be absorbed from the boundary which leads to unnecessary reflection from the uniaxial PML. Hence, an approximate spherical/radial formulation was introduced for the finite-volume time-domain method [12] which applied a local coordinate transformation. This approach has the advantage of avoiding the computation of convolutions and requires only a few additional operations due to the local transformation.
For a two-dimensional transverse-electric case, the update equations of the radial PML based on a Maxwellian absorber [13] take the following form [14]:

$\begin{aligned} \partial_{t} H_{x}= & -\frac{1}{\mu} \partial_{y} E_{z}+\zeta_{r} H_{y} \cos \varphi \sin \varphi-\zeta_{r} H_{x} \sin ^{2} \varphi \\ & -K_{r} \cos \varphi \\ \partial_{t} H_{y}= & \frac{1}{\mu} \partial_{x} E_{z}+\zeta_{r} H_{x} \cos \varphi \sin \varphi-\zeta_{r} H_{y} \cos ^{2} \varphi-K_{r} \sin \varphi\end{aligned}$

$\partial_{t} E_{z}=\frac{1}{\varepsilon}\left[\partial_{x} H_{y}-\partial_{y} H_{x}\right]-\zeta_{r} E_{z}$

$\partial_{t} K_{r}=\frac{1}{\mu}\left[-\zeta_{r} \sin \varphi \partial_{x} E_{z}+\zeta_{r} \cos \varphi \partial_{y} E_{z}\right]$.

The conductivity is determined through the radially dependent parameter $\zeta_{r}$. The angular axis position $\varphi$, defined with respect to the $x$-axis, is a result of the coordinate transformation.

The additionally introduced magnetic polarization variable $K_{r}$ is solved in the differential equation (7d). A method to solve this set of equations in a leap-frog time-stepping scheme for staggered time-domain meshless methods has been introduced in [8]. For the simulations presented in the following, the incoming plane wave (5) is imposed on the PEC and the computational domain is enclosed by the radial PML.

\section{NUMERICAL EXPERIMENT}

To validate the formulation, $2 \mathrm{D}$ numerical experiments are performed considering the scattering from a PEC cylinder. This problem is challenging for the FDTD method, as starcasing effects hinder the convergence of the numerical solutions. For the meshless RPIM scheme, conformal placement of the nodes allows an accurate modeling of the structure. In the following, a convergence study is performed and the impact of the PML thickness is estimated.

\section{A. Mie Scattering}

The scattering from cylindrical objects is a well-known problem in classical electromagnetics with an analytical solution derived by Mie [15]. The scattered transverse electric field at positions $(\rho, \theta)$ from a cylinder with radius $R$ is determined as [16]

$$
E_{s c a t}^{t h}(\rho, \theta)=-E_{0} \sum_{n=-\infty}^{\infty} j^{-n} \frac{J_{n}(k R)}{H_{n}^{(2)}(k R)} H_{n}^{(2)}(k \rho) e^{-j n \theta}
$$

for an incident plane wave of wave number $k$ and amplitude $E_{0}$. The expression contains the Bessel function of the first kind $J_{n}(x)$ and the Hankel function $H_{n}^{(2)}(x)$ of the second kind.

In this paper, a cylinder with a radius of $R=0.2 \mathrm{~m}$ is illuminated by a plane wave pulse with a half-power bandwidth within $f=[2,6] \mathrm{GHz}$. A cylindrical computational domain is created with a radius of $R_{\Omega}=0.5 \mathrm{~m}$, which is clad on the inside with a PML of a thickness corresponding to the maximum simulated wavelength $d=0.15 \mathrm{~m}$. A quadratic conductivity profile is set to a theoretical reflection coefficient 

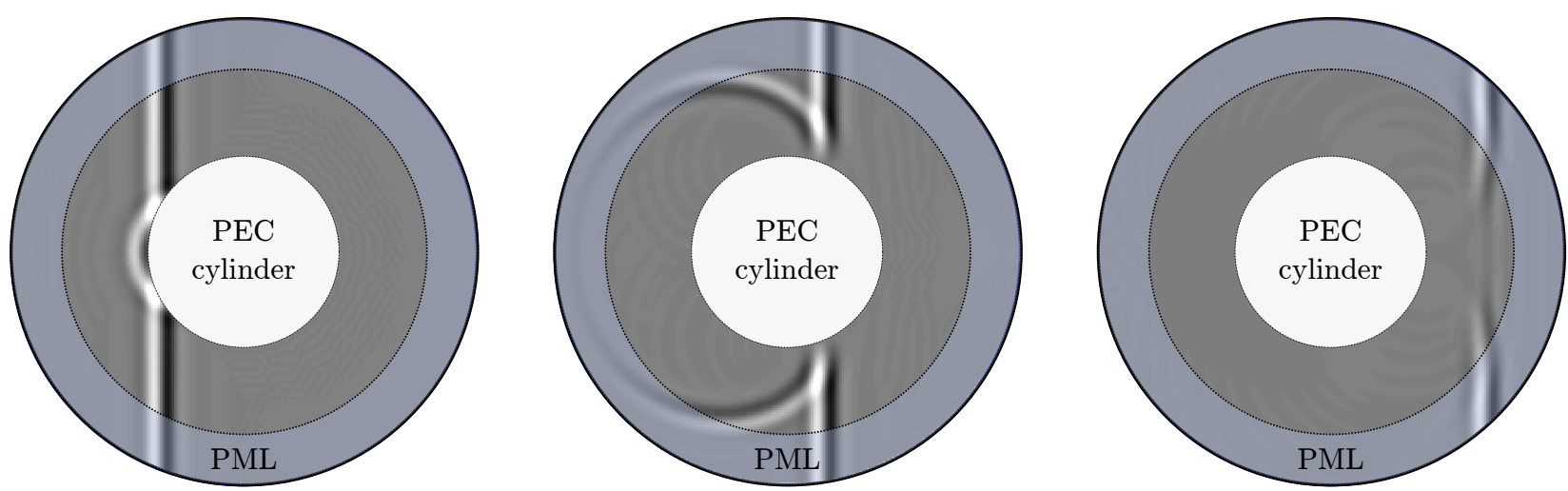

Fig. 1. Plot of the total transverse electric field after 50, 125 and 200 time steps. Scattered fields are absorbed by the radial PML.

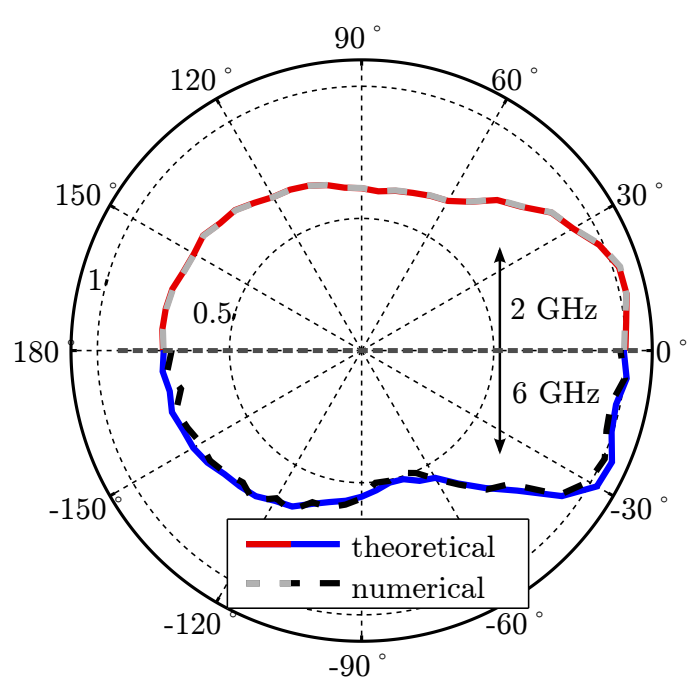

Fig. 2. Total transverse electric field after the Fourier transformation at radially distributed sensor locations for two frequencies $(2 \mathrm{GHz}$ and $6 \mathrm{GHz})$. Due to the symmetry of the results, the lower frequency is shown on the upper half plane and the higher frequency on the lower half plane.

at normal incidence of $\Gamma_{t h}=-80 \mathrm{~dB}$. This parameter corresponds to the accumulated absorption through the layer [11] Transient snapshots of the total simulated electric field for a discretization corresponding to an average node distance of $\Delta x=10 \mathrm{~mm}\left(\lambda_{\min } / 5\right)$ are shown in Fig. 1. The absorption of the scattered field can be clearly observed in the PML. The analytically defined incident plane wave is not absorbed inside the PML, as it is only added for display purposes in the graphs. The simulation time on a standard PC for the whole pulse to propagate through the computational domain is less than $2 \mathrm{~s}$.

For evaluation of the convergence, the scattered electric field is recorded at a number of radially distributed sensor locations (at radius $R_{\text {sensor }}=0.27 \mathrm{~m}$ ) in the near field of the cylinder, and then transformed into the frequency domain. Fig. 2 shows a comparison between the numerical and theoretical (8) scattered field at the edges of the simulated frequency spectrum $(f=[2,6] \mathrm{GHz})$. A very good agreement between the two curves is observed with slightly larger deviations at the higher frequencies, where the discretization becomes very coarse.

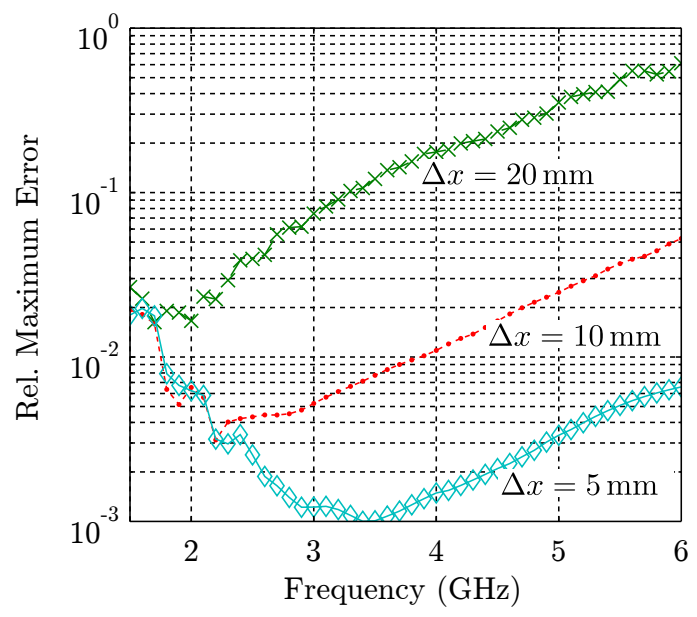

Fig. 3. Convergence of the relative maximum error of the electric field for various node densities.

In the following, a study of the convergence behavior of the relative maximum error over all angles $\theta$

$$
\epsilon(f)=\frac{\max _{\theta}\left|E_{\text {scat }}^{t h}(f, \theta)-E_{\text {scat }}^{\text {num }}(f, \theta)\right|}{\max _{\theta}\left|E_{\text {scat }}^{\text {th }}(f, \theta)\right|}
$$

is performed. This quantifies the error as a function of the frequency for different node densities, i.e. varying discretization in terms of wavelengths.

\section{B. Convergence}

The convergence behavior is investigated here with various node distributions. Starting from a very coarse average node density of $\Delta x=20 \mathrm{~mm}$, which corresponds to $\lambda_{\min } / 2.5$ at the highest frequency of interest, the discretization is refined up to an average node density of $\Delta x=5 \mathrm{~mm}$, which corresponds to a relative density of $\lambda_{\min } / 10$. Fig. 3 shows the relative maximum error (9) over the simulated frequency range. For the relatively coarse discretization considered, low errors down to $\epsilon=10^{-3}$ can be observed.

Two effects are visible: Firstly, for increasing node densities the error decreases as expected. All the curves run in parallel, 


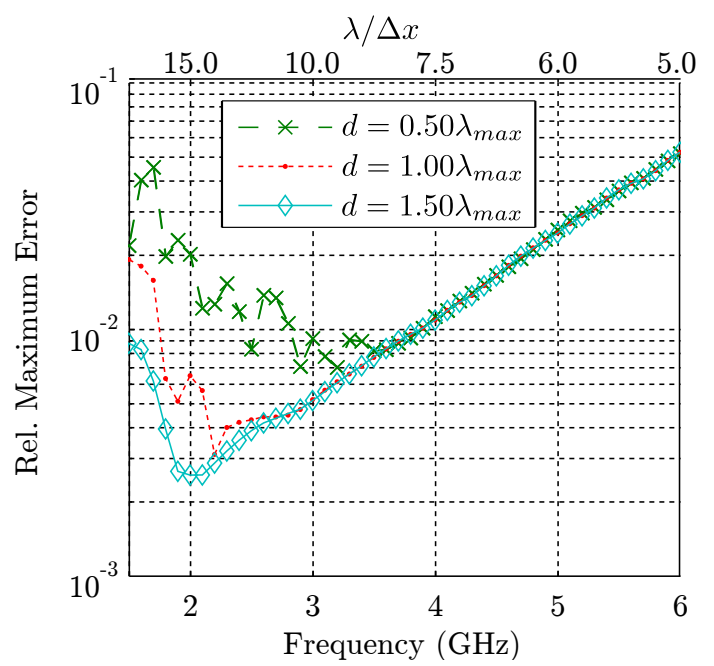

Fig. 4. Relative maximum error for various thicknesses of the PML layer at a discretization of $\Delta x=10 \mathrm{~mm}$.

showing a very quick convergence towards finer discretizations. Secondly, the limitations of the approximate radial PML become visible towards the lower end of the frequency spectrum. Errors introduced as reflections from the PML (domain truncation errors) can be attributed to discretization errors, i.e. the finite number of layers to discretize the conductivity profile and to inaccuracies due to the radius of curvature, which is specific to this type of radial PML [12]. In the following, the first type of truncation error is further investigated.

\section{PML Thickness}

Using a fixed discretization with an average node density of $\Delta x=10 \mathrm{~mm}$, the thickness of the PML is increased to reduce the numerical reflections due to the discretization of the conductivity profile. Starting from a thin PML of a thickness of a quarter of the maximum wavelength $\left(d=0.5 \lambda_{\max }, \sim 7\right.$ node layers), the thickness is increased up to a thickness of $d=1.5 \lambda_{\max }(\sim 22$ node layers $)$. The theoretical reflection coefficient is held constant at $\Gamma_{t h}=-80 \mathrm{~dB}$. The relative maximum error for these thicknesses is shown in Fig. 4. It can be observed that increasing the thickness clearly lowers the reflections from the absorbing boundary, and as a result the simulation accuracy at lower frequencies is increased. In this situation, increasing the thickness from $d=\lambda_{\max }$ (results in Fig. 3) to $d=1.5 \lambda_{\max }$ lowers the breakdown frequency from $2.2 \mathrm{GHz}$ to $1.8 \mathrm{GHz}$

\section{CONCLUSIONS}

A meshless time-domain formulation has been introduced for the scattering from metallic object based on the RPIM. In a scattered field formulation, the source conditions are imposed directly on perfect electric conductors. This implementation can easily be extended to a total field/scattered field formulation. Radial PML have also been implemented to allow for an efficient conformal modeling of cylindrical structures. The Mie scattering from a perfectly electrical conducting cylinder has been used to validate the simulation results. Very good accuracy could be observed at relatively coarse discretizations and relatively low computational effort. The results showed a fast convergence rate, up to a limit where reflections arising from the PML start degrading the accuracy. It has been shown that one way to reduce this effect is increasing the PML thickness, i.e. reducing the conductivity discretization errors. In a next step, the effect of further PML parameters, including the radius of curvature, will be investigated.

\section{ACKNOWLEDGMENT}

C. Fumeaux acknowledges the support of the Australian Research Council (ARC) Future Fellowship funding scheme (under FT100100585).

\section{REFERENCES}

[1] T. Kaufmann, C. Fumeaux, and R. Vahldieck, "The meshless radial point interpolation method for time-domain electromagnetics," in IEEE MTT-S International Microwave Symposium Digest. Atlanta, GA, USA: IEEE, June 2008, pp. 61-65.

[2] Y. Yu and Z. D. Chen, "A 3-D radial point interpolation method for meshless time-domain modeling," IEEE Trans. Microwave Theory and Techn., vol. 57, no. 8, pp. 2015-2020, Aug. 2009.

[3] T. Kaufmann, C. Engström, C. Fumeaux, and R. Vahldieck, "Eigenvalue analysis and longtime stability of resonant structures for the meshless radial point interpolation method in time domain," IEEE Trans. Microwave Theory and Techn., vol. 58, no. 12, pp. 3399-3408, December 2010.

[4] Y. Yu and Z. D. Chen, "An unconditionally stable radial point interpolation method for efficient meshless modeling in time domain," in 25th Annual Review of Progress in ACES, Monterey, USA, March 2009.

[5] X. Chen, Z. Chen, Y. Yu, and D. Su, "An unconditionally stable radial point interpolation meshless method with Laguerre polynomials," IEEE Trans. Antennas and Propagation, vol. 59, no. 10, pp. 3756-3763, Oct. 2011.

[6] Y. Yu and Z. D. Chen, "Meshless RPIM modeling of open-structures using PMLs," in IEEE MTT-S International Microwave Symposium Digest, May 2010, pp. 97-100.

[7] T. Kaufmann, Y. Yu, C. Engström, Z. Chen, and C. Fumeaux, "Recent developments of the meshless radial point interpolation method for time-domain electromagnetics," International Journal of Numerical Modelling: Electronic Networks, Devices and Fields, pp. 1-1, 2012. [Online]. Available: http://dx.doi.org/10.1002/jnm.1830

[8] T. Kaufmann and C. Fumeaux, "Explicit time-stepping scheme for radial perfectly matched layers in staggered meshless methods," in 6th European Conference on Antennas and Propagation (EUCAP) (EuCAP 2012), Prague, Czech Republic, Mar. 2012.

[9] G. R. Liu and Y. T. Gu, An Introduction to Meshfree Methods and their Programming. Dortrecht, The Netherlands: Springer, 2005.

[10] A. Taflove and S. C. Hagness, Computational Electrodynamics: The Finite-Difference Time-Domain Method, 3rd ed. Norwood, MA, USA: Artech House, Inc., June 2005.

[11] J.-P. Bérenger, "Perfectly matched layer (PML) for computational electromagnetics," Synthesis Lectures on Computational Electromagnetics, vol. 2, no. 1, pp. 1-117, 2007.

[12] C. Fumeaux, K. Sankaran, and R. Vahldieck, "Spherical perfectly matched absorber for finite-volume 3-D domain truncation," IEEE Trans. Microwave Theory and Techn., vol. 55, no. 12, pp. 2773-2781, 2007.

[13] R. Ziolkowski, "Time-derivative Lorentz material model-based absorbing boundary condition," IEEE Trans. Microwave Theory and Techn., vol. 45, no. 10, pp. 1530-1535, 2002.

[14] K. Sankaran, C. Fumeaux, and R. Vahldieck, "Uniaxial and radial anisotropy models for finite-volume Maxwellian absorber," IEEE Trans. Microwave Theory and Techn., vol. 54, no. 12, pp. 4297-4304, Dec. 2006.

[15] G. Mie, "Contributions to the optics of turbid media, particularly of colloidal metal solutions," Ann. Phys., vol. 25, no. 3, pp. 377-445, 1908.

[16] S. Narkimelli and H. Ochoa, "Scattering of electromagnetic radiation for a perfect electric conducting cylinder by using multiple angles of polarization," in 42nd Southeastern Symposium on System Theory (SSST) 2010, Mar. 2010, pp. 60-65. 